\title{
Chronic ataluren (PTC124) treatment of nonsense mutation cystic fibrosis
}

\author{
M. Wilschanski*, L.L. Miller", D. Shoseyov*, H. Blau`, J. Rivlin ${ }^{+}$, M. Aviram ${ }^{\S}$, \\ M. Cohen*, S. Armoni*, Y. Yaakov*, T. Pugatch*, M. Cohen-Cymberknoh*, \\ N.L. Miller" ${ }^{\#}$ A. Reha ${ }^{\#}$, V.J. Northcutt ${ }^{\#}$, S. Hirawat" ${ }^{\#}$ K. Donnelly", G.L. Elfring ${ }^{\#}$, \\ T. Ajayi" and E. Kerem*
}

ABSTRACT: In a subset of patients with cystic fibrosis (CF), nonsense mutations (premature stop codons) disrupt production of full-length, functional CF transmembrane conductance regulator (CFTR). Ataluren (PTC124) allows ribosomal readthrough of premature stop codons in mRNA.

We evaluated drug activity and safety in patients with nonsense mutation CF who took ataluren three times daily (morning, midday and evening) for 12 weeks at either a lower dose (4, 4 and $\left.8 \mathrm{mg} \cdot \mathrm{kg}^{-1}\right)$ or higher dose $\left(10,10\right.$ and $\left.20 \mathrm{mg} \cdot \mathrm{kg}^{-1}\right)$.

The study enrolled 19 patients (10 males and nine females aged 19-57 yrs; dose: lower 12, higher seven) with a classic CF phenotype, at least one CFTR nonsense mutation allele, and an abnormal nasal total chloride transport. Both ataluren doses were similarly active, improving total chloride transport with a combined mean change of $-5.4 \mathrm{mV}(\mathrm{p}<0.001)$, and on-treatment responses (at least $-5 \mathrm{mV}$ improvement) and hyperpolarisations (values more electrically negative than $-5 \mathrm{mV})$ in $61 \%(\mathrm{p}<0.001)$ and $56 \%(\mathrm{p}=0.002)$ of patients. CFTR function was greater with time and was accompanied by trends toward improvements in pulmonary function and CF-related coughing. Adverse clinical and laboratory findings were uncommon and usually mild.

Chronic ataluren administration produced time-dependent improvements in CFTR activity and clinical parameters with generally good tolerability.

KEYWORDS: Adult, cough, cystic fibrosis transmembrane conductance regulator, investigational drug, nasal potential difference, nonsense mutation

ystic fibrosis (CF) is an autosomal recessive inherited disorder that results from mutations leading to dysfunction in the CF transmembrane conductance regulator (CFTR), a chloride channel located at the apical surface of epithelial cells [1]. In patients with CF, disruption of CFTR-mediated ion and water transport leads to abnormally viscous secretions that obstruct the lungs, pancreas, biliary tree, intestine and other organs [2]. Pulmonary manifestations are prominent; patients commonly exhibit airway inflammation, infection and obstruction, leading to progressive lung damage $[3,4]$. The most prevalent symptom of the disease is coughing [5-7]. Ultimately, progressive lung dysfunction leads to respiratory failure and death in most patients $[8,9]$. Current supportive treatment options (e.g. inhaled mucolytics, antibiotics and osmotic agents) provide benefit [10-12], but there is no approved therapy to correct CFTR production and function.

A nonsense mutation in DNA generates a UGA, UAG or UAA premature stop codon within the protein-coding region of the corresponding mRNA. Premature stop codons result in disease when they disrupt ribosomal translation of the mRNA for a critical protein, such as the CFTR. Nonsense mutations in the CFTR gene cause CF in $5-10 \%$ of patients worldwide [13] and in $>60 \%$ of CF patients in Israel [14]. Patients with nonsense mutation CF generally exhibit a severe CF phenotype [15-17].

Ataluren (PTC124) is an investigational, orally bioavailable, small molecule drug designed to overcome the adverse effects of a nonsense mutation by inducing selective ribosomal readthrough of mRNA containing a premature stop codon and allowing translation of a full-length protein [18]. Preclinical evaluation indicated that ataluren generates functional CFTR in a nonsense mutation animal model of CF [19]. In a prior short-term proof-of-concept trial in nonsense mutation $\mathrm{CF}$, we administered ataluren in two 14-day treatment courses separated by a 14-day washout period [20]. Drug administration regimens comprised morning, midday and evening

\section{AFFILIATIONS}

*Hadassah Hebrew University Hospital, Jerusalem,

"Schneider Children's Hospital,

Petach Tikvah,

${ }^{+}$Carmel Hospital, Haifa,

${ }^{\S}$ Soroka Medical Center, Be'er Sheva, Israel, and

\#PTC Therapeutics, South Plainfield, NJ, USA.

CORRESPONDENCE

M. Wilschanski

Paediatric Gastroenterology Hadassah University Hospital Mount Scopus POB 24035 Jerusalem

91240

Israel

E-mail: michaelwil@hadassah.org.il

Received:

July 282010

Accepted after revision:

Oct 222010

First published online:

Jan 132011 
doses of ataluren; in the first course, patients received a lower dose level of 4,4 and $8 \mathrm{mg} \cdot \mathrm{kg}^{-1}$ and in the second course they received a higher dose level of 10,10 and $20 \mathrm{mg} \cdot \mathrm{kg}^{-1}$ of ataluren. Our results showed that short-term oral administration of ataluren as a nonsense mutation suppression therapy was generally well tolerated and improved the epithelial electrophysiological abnormalities caused by CFTR dysfunction.

We designed this study to extend those observations by evaluating the activity and safety of 12 weeks of continuous ataluren treatment in the same patients who had participated in the short-term study. The primary objective was to assess pharmacodynamic activity as determined by changes in nasal transepithelial potential difference (NPD). Secondary objectives included assessment of changes in pulmonary function and cough rate, evaluation of safety and study drug compliance, and characterisation of ataluren plasma exposures over time. We have previously presented preliminary data from this study in abstract form [21, 22].

\section{METHODS \\ Ethics}

The institutional ethics committee and the Israeli Ministry of Health approved the study protocol. Each participant provided signed informed consent prior to conduct of any study procedures. We conducted the trial according to Good Clinical Practice and listed the study on www.clinicaltrials.gov (identifier NCT00351078).

\section{Eligibility criteria}

Patients were adults $\geqslant 18$ yrs of age who had participated in the previous phase 2 trial [20]. All patients had phenotypic evidence of CF and two disease-causing CFTR mutations, with at least one being a nonsense mutation as determined by gene sequencing (Ambry Genetics, Inc., Aliso Viejo, CA, USA). At the study baseline, all patients had a sweat concentration outside of the normal range (value $>40 \mathrm{mEq} \cdot \mathrm{L}^{-1}$ in sweat collected by pilocarpine iontophoresis), abnormal total chloride transport as assessed by NPD (value more electrically positive than $-5 \mathrm{mV}$ during successive nasal perfusions with chloride-free amiloride followed by chloride-free amiloride with isoproterenol) $[23,24]$, a forced expiratory volume in $1 \mathrm{~s}$ $($ FEV 1$) \geqslant 40 \%$ of predicted, and an oxygen saturation of $\geqslant 92 \%$ on room air. Exclusion criteria included pre-existing or ongoing clinically significant illnesses or complications of CF, serum bilirubin greater than normal limits, serum transaminase values $\geqslant 2$ times normal limits, serum creatinine $>1.5$ times the upper limits of normal, or use of systemic or inhaled aminoglycosides within 14 days prior to starting ataluren. Patients could take stable regimens of other inhaled drugs and oral pancreatic enzymes. Female patients could not be pregnant or breastfeeding.

\section{Study treatment}

PTC Therapeutics, Inc, South Plainfield, NJ, USA provided ataluren as vanilla-flavoured granules in aluminum foil sachets. We instructed patients to mix the granules with plain water, apple juice or milk, to make a suspension for oral administration. Patients were to take ataluren three times per day (morning, midday and evening) for 84 days (12 weeks) of continuous daily treatment followed by 28 days ( 4 weeks) of follow-up without study drug. While ataluren can be given either fasting or fed, we recommended ingestion within $30 \mathrm{~min}$ after a meal based on phase 1 pharmacokinetic data indicating that plasma concentrations were better sustained when the drug is taken with food [25]. We based allocation to ataluren dose level on the magnitude of the change in total chloride transport we had observed in the prior short-term study. If a patient's best total chloride transport improvement had occurred at the lower dose level $\left(4 \mathrm{mg} \cdot \mathrm{kg}^{-1}\right.$ in the morning, $4 \mathrm{mg} \cdot \mathrm{kg}^{-1}$ at midday and $8 \mathrm{mg} \cdot \mathrm{kg}^{-1}$ in the evening) in the prior study, then the patient was to be assigned to the 4-, 4- and 8$\mathrm{mg} \cdot \mathrm{kg}^{-1}$ dose level in this study. If a patient's best total chloride transport improvement had occurred at the higher dose level $\left(10 \mathrm{mg} \cdot \mathrm{kg}^{-1}\right.$ in the morning, $10 \mathrm{mg} \cdot \mathrm{kg}^{-1}$ at midday and $20 \mathrm{mg} \cdot \mathrm{kg}^{-1}$ in the evening) in the prior study, then the patient was to be assigned to the $10-, 10-$ and $20-\mathrm{mg} \cdot \mathrm{kg}^{-1}$ dose level in this study.

\section{Study assessments}

We assessed NPD in each nostril using standardised methods [26]. Warmed solutions were perfused through a nasal catheter while a voltage tracing was recorded. Solutions comprised Ringer's lactate to obtain a basal potential difference, amiloride to inhibit the epithelial sodium channel, a chloride-free of sodium and calcium gluconate with amiloride to induce an electrogenic chloride gradient, and isoproterenol in chloridefree gluconate with amiloride to stimulate CFTR secretion of chloride ions from the epithelium. The chloride transport during the perfusions of gluconate (intrinsic chloride transport) and isoproterenol (stimulated chloride transport) constituted the total CFTR effect (total chloride transport), the primary measure of the study. For each parameter, we analysed the average of the available values from the left and right nostrils. We had NPD tracings independently reviewed by an external expert (M. Sinaasappel, Rotterdam, the Netherlands), blinded as to study time point; the reported values are those of the independent reviewer.

FEV1 and forced vital capacity (FVC) were assessed according to American Thoracic Society/European Respiratory Society guidelines [27, 28]. We quantified cough using the VivoMetrics LifeShirt $^{\mathrm{TM}}$ (VivoMetrics, Ventura, CA, USA) as previously described [7]. This device incorporates chest-wall, motionsensing transducers; electrocardiographic electrodes, a unidirectional throat microphone, and a three-axis accelerometer into a lightweight vest; integrated input from the motion sensors and microphone provides an analysis of cough rate [29]. Following each clinic visit, patients were to wear the LifeShirt vest for $24 \mathrm{~h}$ at home during normal activities of daily living. Adverse events and laboratory abnormalities were assessed using conventional clinical techniques. We quantified ataluren compliance based on data from patient-completed compliance logs. Ataluren plasma concentrations were assayed using a validated bioanalytical method [25].

We obtained activity and safety assessments at the beginning of treatment, every 28 days during treatment and 28 days after the end of treatment. Before and $3 \mathrm{~h}$ after the morning ataluren dose on days 1, 28, 56 and 84, we collected blood samples for determination of ataluren plasma concentrations. In addition, we performed post-study safety assessments at $\sim 6$-month intervals. 


\section{Statistics}

The sample size was bounded by the number of patients $(n=23)$ that had been enrolled to the prior phase 1 study. We predefined total chloride transport response (at least $-5 \mathrm{mV}$ improvement) and hyperpolarisation (equal to or more electrically negative than $-5 \mathrm{mV}$ ) based on past experience $[23,24,30-32]$. We intended to enrol $\geqslant 18$ evaluable patients in order to achieve a power $\geqslant 0.90$ (exact binomial test, one-sided $\alpha=0.05$ ) to reject a $<10 \%$ spontaneous total chloride transport response or hyperpolarisation proportion (null hypothesis) in favour of a $\geqslant 40 \%$ pharmacologically induced response or hyperpolarisation proportion (alternative hypothesis). With a sample size of 18 patients we also had power $>0.90$ (paired $t$ test, two-sided $\alpha=0.05$ ) to detect a change in total chloride transport of similar magnitude and variability, as was observed in our prior ataluren study [20].

We used the standard equation to determine body mass index. Similarly, pulmonary function values were calculated as per cent predicted values for age, height and sex [33]. On-study relative changes were expressed using per cent predicted values at baseline for reference. LifeShirt data were analysed using device-specific software with predefined algorithms that counted coughs and differentiated between wake and sleep status [7]. Consistent with past methods [7, 29], a cough was defined as $\geqslant 1$ tussive blasts, each separated by $<1 \mathrm{~s}$ and occurring within the same expiratory breath. We determined waking and sleeping cough rates by dividing the number of coughs within a wake or sleep period by the time spent wearing the LifeShirt during that period. We characterised individual cough duration in ms and cough intensity as the amplitude of the cough signal measured in $\mathrm{mV}$.

We evaluated activity variables in all patients with sufficient baseline and on-study measurements for the test of interest. Safety and compliance were assessed in all patients who received at least one dose of ataluren. We assessed the proportion of patients with a total chloride transport response relative to the null-hypothesis response rate of $10 \%$ using a one-sided exact binomial test. We analysed changes in NPD parameters, relative pulmonary function and cough rates using paired t-tests. Changes in the proportions of patients with hyperpolarised total chloride transport values were assessed using McNemar's test. Effects by dose were assessed using an unpaired t-test for best on-treatment changes and Fisher's exact test for response and hyperpolarisation proportions. As was specified in the protocol, we considered p-values $\leqslant 0.05$ to be statistically significant. Adverse events and laboratory abnormalities were coded using the Medical Dictionary for Regulatory Activities (www.meddramsso.com) and worst severity was graded using the Common Terminology Criteria for Adverse Events, version 3.0 (http://ctep.cancer.gov/ forms). We calculated compliance for each patient as the proportion of actual relative to planned ataluren doses. Standard regression techniques were employed.

\section{RESULTS}

\section{Patients}

Of the 23 patients who participated in the preceding phase $2 \mathrm{a}$ study [20], four could not enrol in this extension study; two had time constraints precluding participation and two were not eligible (one developed a CF exacerbation during screening, and one had an FEV1 $<40 \%$ and required chronic oxygen supplementation). Of the 19 patients who agreed to participate in this trial, 12 were allocated to the lower $4-, 4$ - and $8-\mathrm{mg} \cdot \mathrm{kg}^{-1}$ dose level and seven patients to the higher 10-, 10- and $20-\mathrm{mg} \cdot \mathrm{kg}^{-1}$ dose level. One patient receiving 4,4 and $8 \mathrm{mg} \cdot \mathrm{kg}^{-1}$ had a grade 1 elevation in creatine on day 52; he elected to withdraw from the study despite investigator encouragement to continue with study participation. One patient receiving 10,10 and $20 \mathrm{mg} \cdot \mathrm{kg}^{-1}$ developed distal ileal obstruction syndrome (DIOS) on day 80 ; she did not resume ataluren after the event because the end of the planned treatment period was day 84 . Thus, 17 patients completed the planned 84 days (12 weeks) of study drug treatment.

Baseline patient characteristics defined a population with typical characteristics for adults with nonsense mutation $\mathrm{CF}$ (table 1). The majority of patients were young adults. Consistent with the autosomal genetics of the disease, similar proportions of males and females participated, although there were more males receiving the lower ataluren dose level. For all patients, sweat chloride values and nasal total chloride transport values were abnormal. Spirometry revealed that most patients had substantial obstructive pulmonary dysfunction, with 14 (78\%) patients having abnormal FEV1 values (i.e. $<90 \%$ pred). Chronic pathological airway infection was nearly universal. Most patients required pancreatic enzyme replacement therapy for exocrine pancreatic insufficiency and several needed insulin for control of CF-related diabetes. Mild to moderate (grade 1 to 2) liver enzyme abnormalities suggesting CF-related hepatobiliary disease were also evident in a few subjects. Three nonsense mutation genotypes were represented, with W1282X being the most common. For five patients, nonsense mutations were present on both alleles. The UGA premature stop codon type predominated. The minimum period from the last ataluren dose in the prior phase 2a study to the first dose in this study was $>8$ months in all patients.

\section{Nasal transepithelial potential difference}

Considering the enrolment of 19 patients and the plan to assess NPD at five study visits (one at baseline, three on study and one at follow-up), we intended to perform a total of 95 NPD evaluations. Of these, three tests were missed because one patient withdrew from the study and four tests were not completed for other reasons (two due to rhinitis and two due to upper respiratory tract infection). Of the remaining 88 NPD assessments, recordings in both nostrils were available from 58 $(66 \%)$ time points and recordings from one nostril were available from 30 (34\%) time points. Reasons for not obtaining readings in both nostrils related to chronic conditions (e.g. deviated septum) or acute factors (e.g. rhinitis and nasal bleeding) that prevented adequate testing in one of the nostrils.

We predefined a response as an on-treatment improvement of least $-5.0 \mathrm{mV}$ from baseline in total chloride transport. As summarised based on the magnitude of the best on-study total chloride transport improvements for each patient (fig. 1), responses were observed in seven out of 11 (64\%, 90\% CI 35$86 \%$; $<<0.001)$ evaluable patients receiving the lower ataluren dose and in four out of seven (57\%, 90\% CI 23-87\%; p<0.001) evaluable patients receiving the higher ataluren dose. When considering all evaluable patients across both dose levels, the response rate was 11 out of $18(61 \%, 90 \%$ CI 39-80\%; p < 0.001$)$. 


\section{TABLE 1 Baseline patient characteristics}

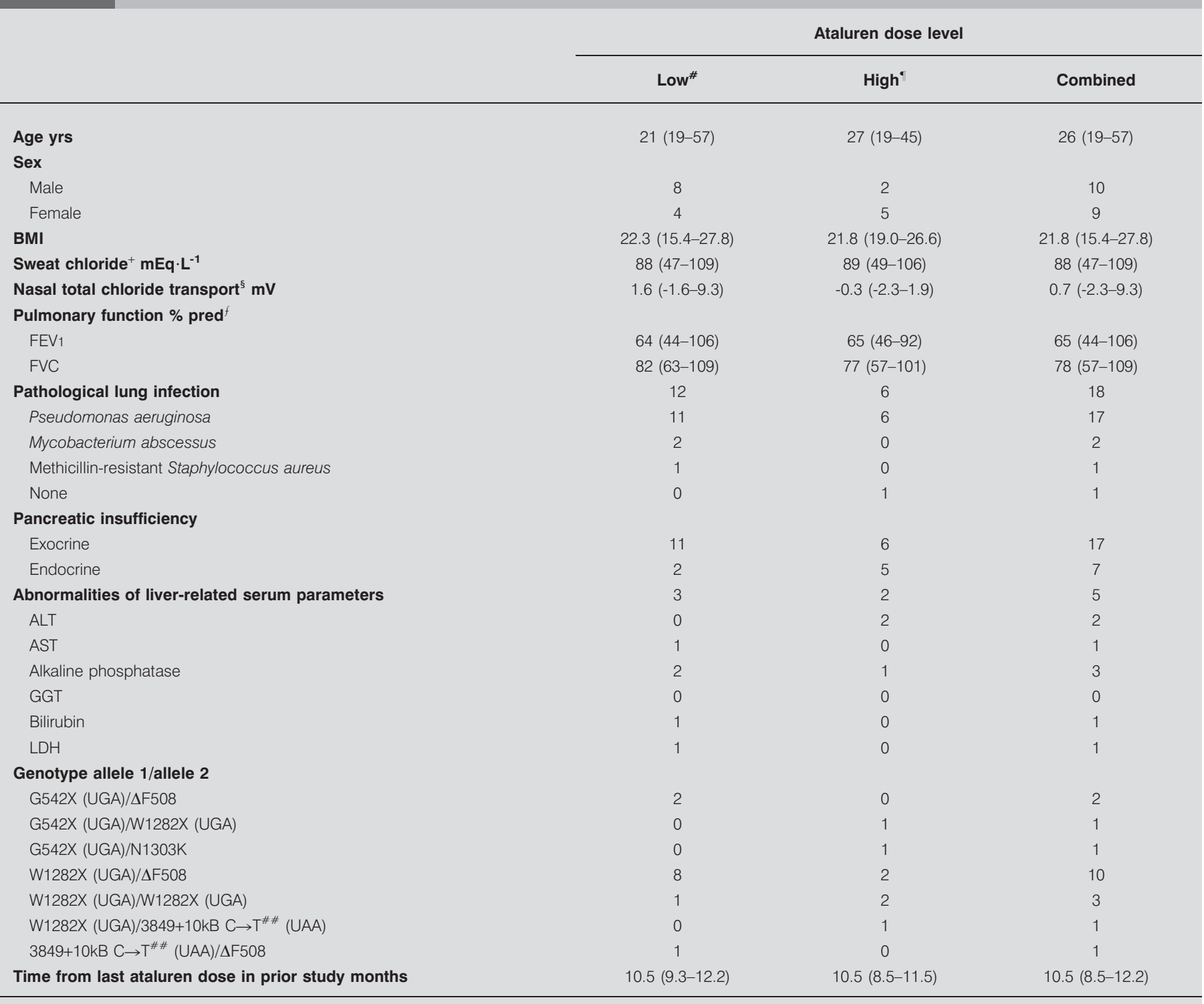

Data are presented as $\mathrm{n}$ or median (range). BMI: body mass index; \% pred: \% predicted; FEV1: forced expiratory volume in $1 \mathrm{~s}$; FVC: forced vital capacity; ALT: alanine aminotransferase; AST: aspartate aminotransferase; GGT: gamma glutamyl transferase; LDH: lactate dehydrogenase. ${ }^{\#}: 4,4$ and $8 \mathrm{mg} \cdot \mathrm{kg}^{-1}, \mathrm{n}=12 ;{ }^{\bullet}: 10,10$ and $20 \mathrm{mg} \cdot \mathrm{kg}^{-1}, \mathrm{n}=7$; $^{+}$: upper limit of normal $40 \mathrm{mEq} \cdot \mathrm{L}^{-1} ;{ }^{\text {s: }}$ upper limit of normal $-5 \mathrm{mV}[23,24]$; ${ }^{\text {: }}$ based on normative data for age, sex and height [33]; ${ }^{\# \#}$ : mutation results in an elongated mRNA containing an in-frame premature UAA stop codon [34].

Based on these data, the primary hypothesis of the study was satisfied, i.e. a low response rate of $<10 \%$ that might be due to random fluctuations in total chloride conductance was excluded with substantial confidence. There was no statistically significant difference in response rate between the two dose levels ( $p=1.0$, Fisher's exact test). Ataluren treatment was associated with an on-treatment total chloride response in both patients with the G542X and W1282X mutations. Surprisingly, the one evaluable patient with the $3849+10 \mathrm{kB} \mathrm{C} \rightarrow$ T mutation, who had responded in the prior short-term study [20], did not meet criteria for response in this study. An examination of the data in the five patients who had nonsense mutations on both alleles did not suggest that these patients were more likely to have an improvement in total chloride transport than patients who had a nonsense mutation on only one allele.

When considering mean total chloride transport values at each dose level and for both dose groups together, ataluren induced statistically significant improvements (fig. 2). Changes were time-dependent, with progressively better mean values as treatment continued. Mean improvements at the end of treatment on day 84 were $-6.8 \mathrm{mV}(\mathrm{p}=0.004)$ for those receiving the lower ataluren dose and were $-3.4 \mathrm{mV}$ $(p=0.025)$ for those receiving the higher ataluren dose, resulting in a mean change for both dose levels combined of $-5.4 \mathrm{mV}(\mathrm{p}<0.001)$. Changes in total chloride transport were 


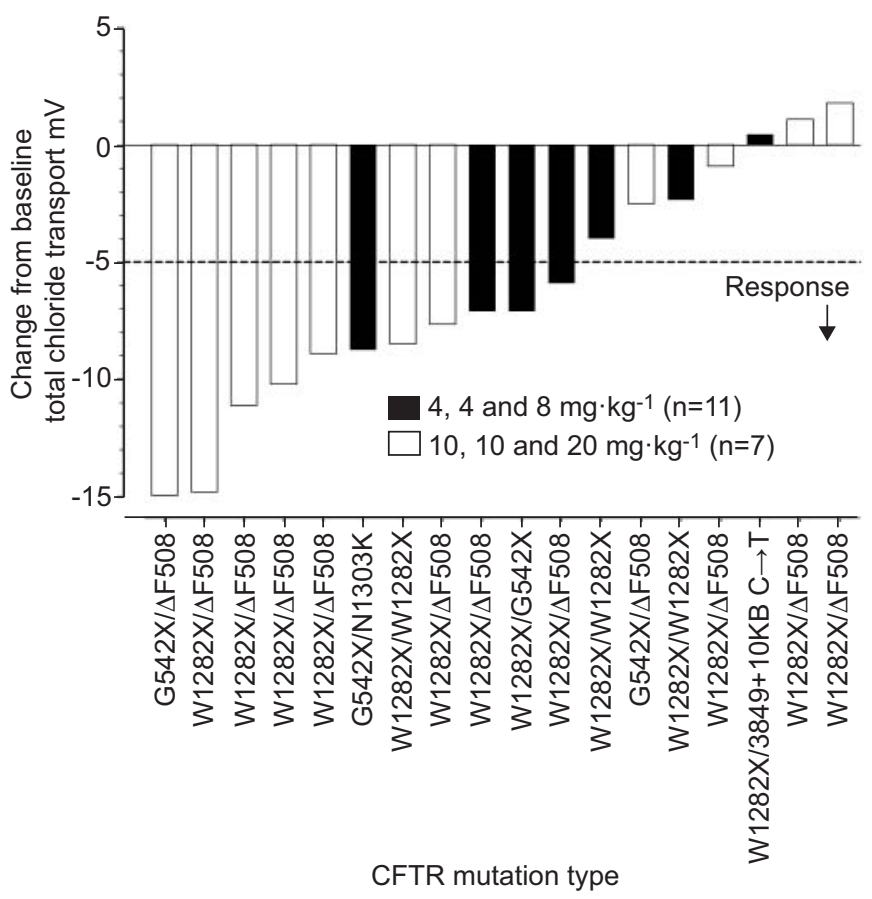

FIGURE 1. Individual best total chloride transport changes. CFTR: cystic fibrosis transmembrane conductance regulator.

due to changes in both intrinsic and stimulated chloride transport values; respective mean improvements in intrinsic and stimulated chloride transport on day 84 were $-5.1 \mathrm{mV}$ $(p=0.015)$ and $-1.7 \mathrm{mV}(p=0.029)$ for those receiving the lower ataluren dose, and were $-3.0 \mathrm{mV}(\mathrm{p}=0.037)$ and $-0.4 \mathrm{mV}$ $(p=0.736)$ for those receiving the higher ataluren dose. Respective mean intrinsic and stimulated chloride transport changes for both dose levels together were $-4.2 \mathrm{mV}(\mathrm{p}=0.002)$ and $-1.2 \mathrm{mV}(\mathrm{p}=0.080)$. Dose dependency was not obvious in these analyses; unpaired t-tests indicated no statistically significant differences between the dose groups for changes in total $(p=0.17)$, intrinsic $(p=0.35)$ or stimulated $(p=0.34)$

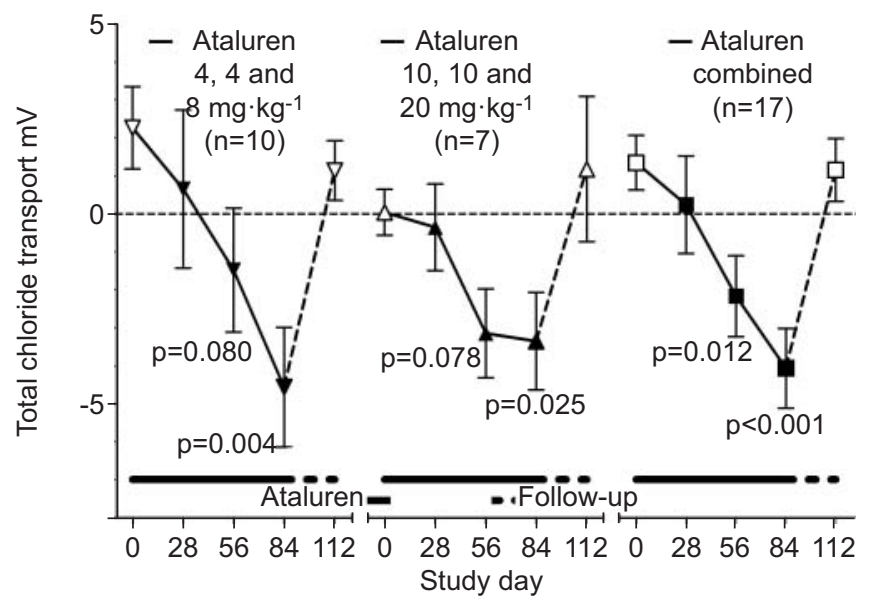

FIGURE 2. Total chloride transport. Data are presented as mean \pm SEM. $\mathrm{p}$ values from paired t-test comparing on-study time point versus corresponding baseline. chloride transport by day 84 . We did not observe significant improvements in basal potential difference or amiloride response values (fig. 3) or meaningful correlations between changes in total chloride transport and changes in basal potential difference or amiloride response values.

An examination of individual patient values (fig. 4) confirmed that all patients had abnormal total chloride transport at baseline and that ataluren treatment induced statistically significant improvements in the proportions of patients with hyperpolarised total chloride transport values (where hyperpolarisation was predefined as an on-treatment value equal to or more electrically negative than $-5.0 \mathrm{mV}$ ). Altogether, 10 of 18 $(56 \%)$ evaluable patients had a total chloride transport hyperpolarisation at one time point during ataluren treatment across both dose levels. Confirming the evaluation of mean changes (fig. 2), changes in individual values were generally time dependent, with progressive improvements with increasing duration of ataluren treatment. No difference in total chloride transport hyperpolarisation by dose was apparent $(\mathrm{p}=1.0$, Fisher's exact test $)$.

Rather than using the mean NPD values obtained from both nostrils (as in the primary analyses), we also analysed outcome
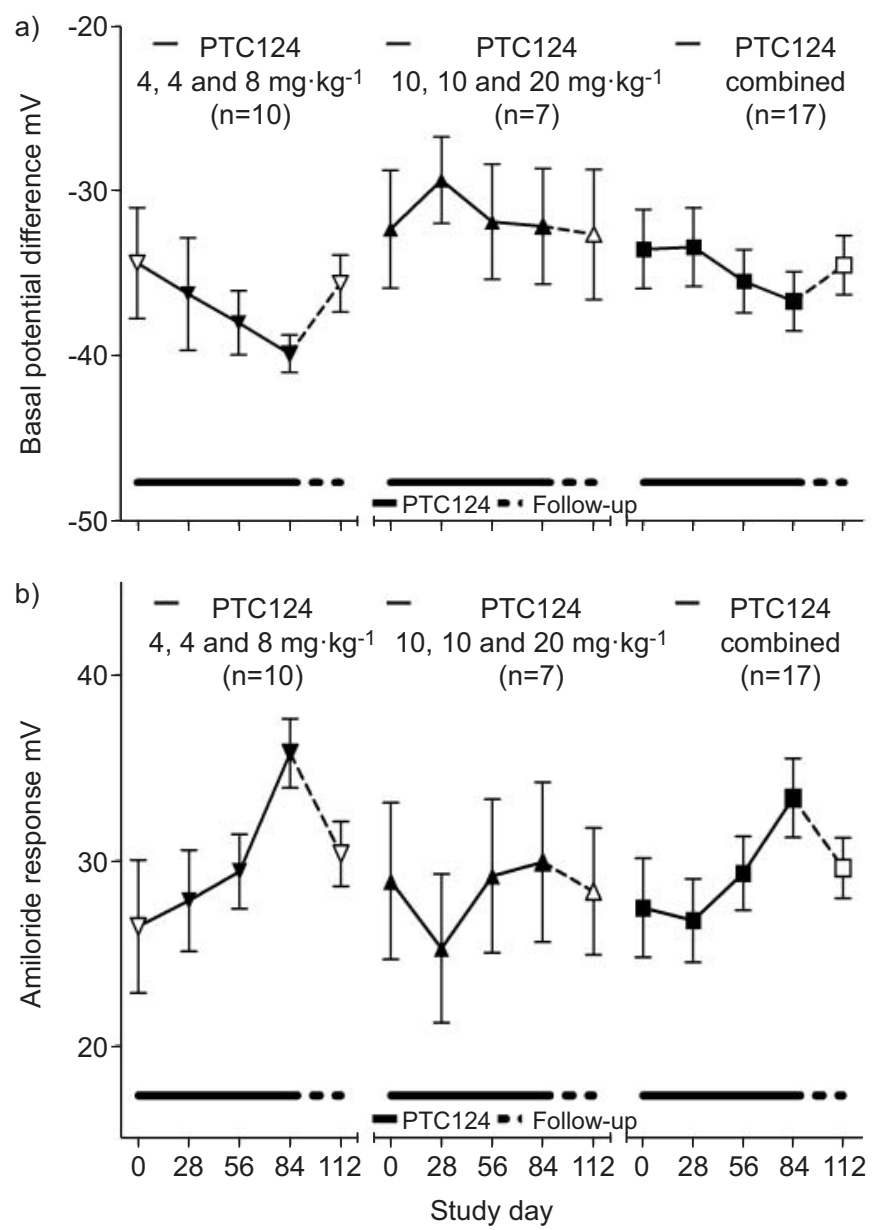

FIGURE 3. a) Basal potential difference and b) amiloride response. Data are presented as mean \pm SEM. $p$-values from paired t-test comparing on-study time point versus corresponding baseline. 
a)

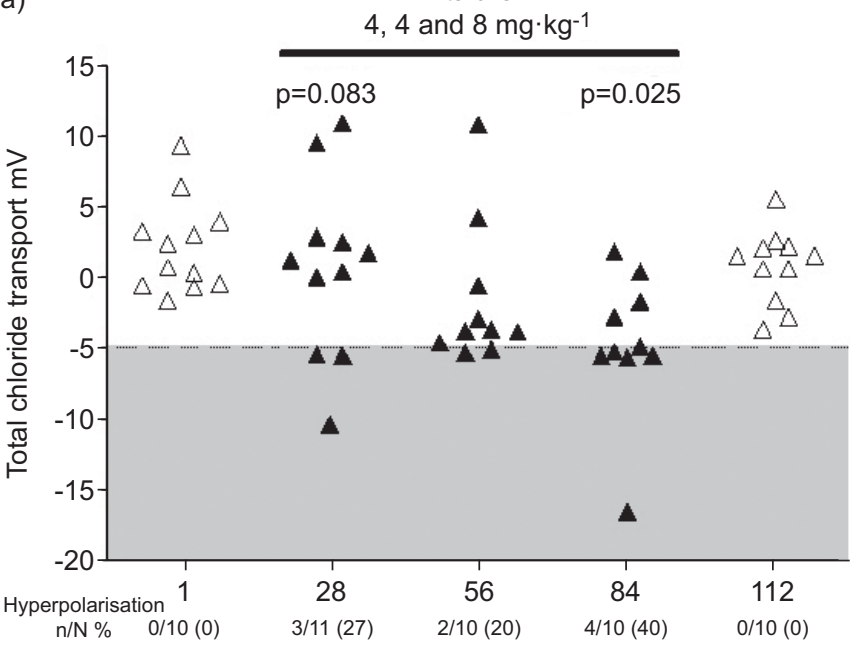

Study day

c)

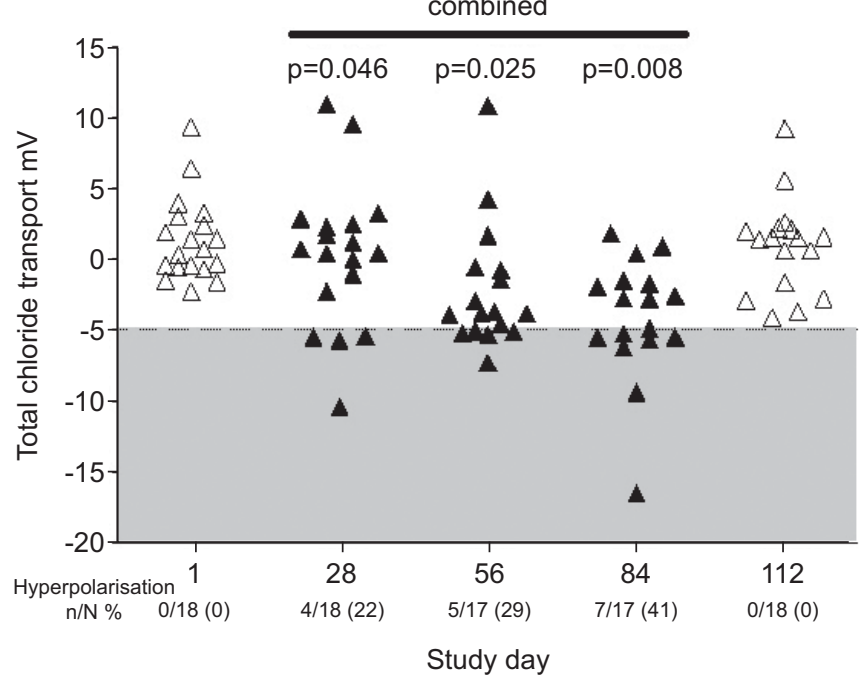

b)

Ataluren
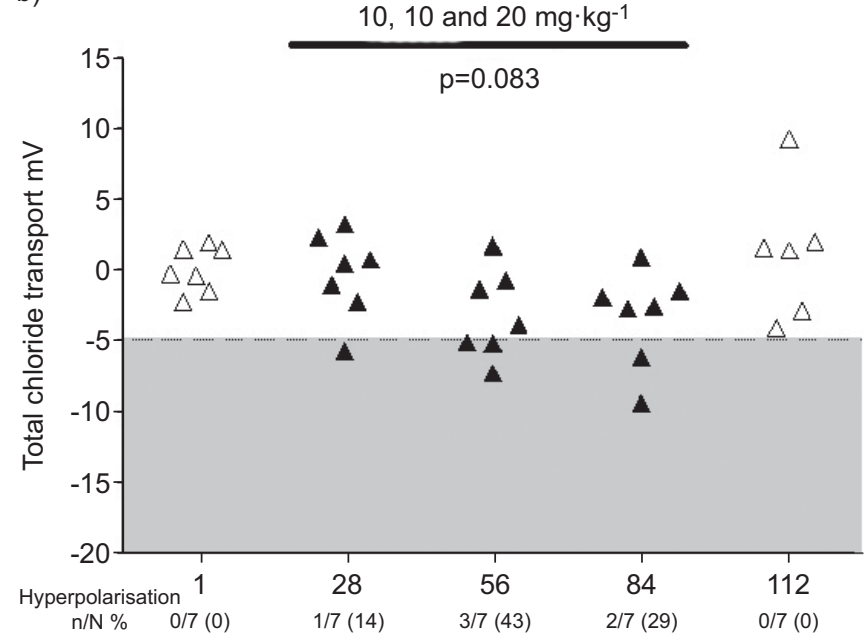

Study day

FIGURE 4. Individual total chloride transport values. p-values from McNemar's test comparing on-study time point versus corresponding baseline.

considering the single best (most hyperpolarised) total chloride transport value available at each visit; the findings of this sensitivity analysis confirmed the pattern and statistical significance of the primary NPD findings (data not shown). Analyses of total chloride response, change and hyperpolarisation by age, sex and baseline total chloride transport value revealed no significant influence of these patient factors on NPD outcomes (data not shown).

\section{Pulmonary function assessment}

Pulmonary function tests were performed at baseline and ontreatment in 18 patients during the course of the study (fig. 4). Changes were not statistically significant; however, the aggregate mean value moved higher through day 84 for the combined dose groups (with changes of $4.5 \%$ and $3.5 \%$ for relative per cent predicted FEV1 and FVC, respectively). Changes in lung function moved in general correspondence with changes in total chloride transport values over the same period (fig. 5).
With cessation of ataluren, mean changes reverted toward baseline by day 112 .

\section{Cough quantitation}

Altogether, 18 patients were evaluable for cough quantitation. For these patients, cough data were collected at every visit (one at baseline, three on treatment and one at follow-up); the median (range) duration of recording was 24 (6-26) h; of these, 15 (5-20) h were recorded as waking and $9(0-15) \mathrm{h}$ were recorded as sleeping. Of the 90 evaluable assessments, 86 of 90 $(96 \%)$ included at least $20 \mathrm{~h}$ worth of data; of the remaining four assessments, two included at least $12 \mathrm{~h}$ of data. At baseline, patients had a mean (range) of $734(324-1,569)$ coughs per day. As expected, baseline mean (range) waking cough rates of 43 (20-82) coughs per hour markedly exceeded sleeping cough rates of 2 (0.1-7) coughs per hour. By day 84, ataluren was associated with reductions in waking cough frequency of $21 \%$ and $25 \%$ in the lower and higher ataluren 
dose groups, respectively (fig. 6); the overall reduction of $23 \%$ for the combined dose groups was statistically significant $(p=0.006$, paired $t$-test). For the combined groups, there was also a time-dependent decrease in median cough duration that achieved greatest significance $(\mathrm{p}=0.007$, paired $\mathrm{t}$-test) at day 84; cough intensity was not significantly changed (data not shown). Sleeping cough rates remained low and generally unaltered throughout the study.

\section{Drug compliance and safety}

There were no physician-prescribed dose interruptions or reductions. Median (range) patient compliance was $96 \%$ (91-102\%) for the 4-, 4- and $8-\mathrm{mg} \cdot \mathrm{kg}^{-1}$ dose regimen and $99 \%$ (78-99\%) for the $10-, 10-$ and $20-\mathrm{mg}^{\circ} \mathrm{kg}^{-1}$ dose regimen. With both regimens, planned doses were only infrequently missed $(\sim 4 \%)$ or reduced $(<0.2 \%)$ by the patients.

We recorded all treatment-emergent adverse events and laboratory abnormalities regardless of their potential relationship to ataluren therapy (table 2). Most adverse events were mild (grade 1) or moderate (grade 2) in severity and showed no obvious dose-dependent increase in frequency or severity. Three patients had grade 1 dysuria without associated urinary abnormalities; ataluren was continued and symptoms abated with hydration after periods of 2, 2 and 20 days. One patient had a transient ischaemic attack while coughing. This event
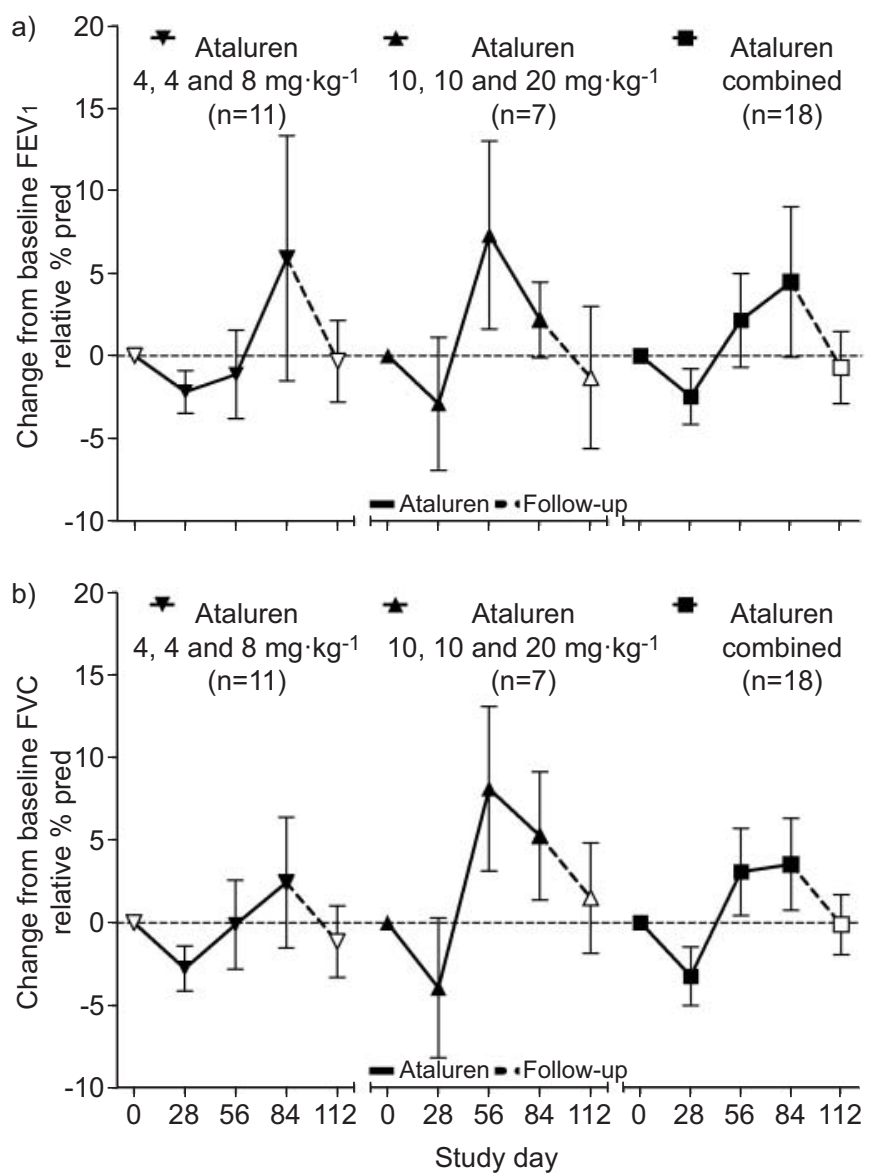

FIGURE 5. Change in mean pulmonary function values. Data are presented as mean \pm SEM. FEV1: forced expiratory volume in $1 \mathrm{~s}$; FVC: forced vital capacity

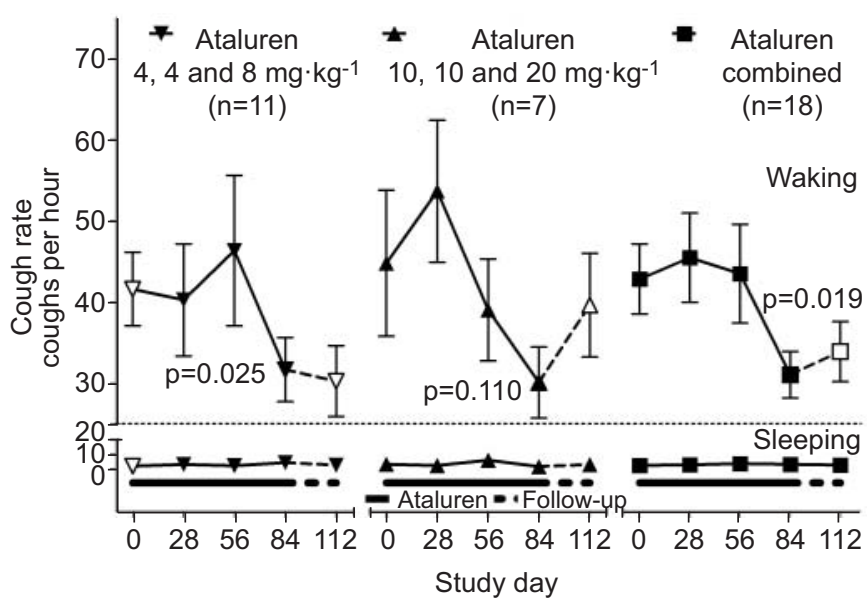

FIGURE 6. Waking and sleeping cough rates. Data are presented as mean \pm SEM. $p$-values from paired t-test comparing on-treatment time point versus corresponding baseline.

was not considered ataluren-related given a past history of such events in this patient and the fact that cough-induced hemiplegic migraine or paradoxical embolisation are known complications of CF [35-37]. Serious adverse events requiring hospitalisation included fungal infection of a central venous catheter, DIOS and a CF exacerbation.

Treatment-emergent laboratory abnormalities were infrequent (table 2). We observed grade 2 or 3 elevation in serum glucose in several patients; this finding appeared to be a CF-related phenomenon in patients with a past history of hyperglycaemia. In nonclinical studies and clinical trials in healthy volunteers, substantially higher ataluren doses than those used in this study have not caused perturbations in glucose metabolism $[18,25]$. Three patients had grade 1 increases in creatinine; in two patients these abnormalities were each ascribed to DIOS and gentamicin administration while in one patient a grade 1 elevation abated with planned cessation of ataluren on day 84 . Occasional sporadic fluctuations in serum aminotransferase values were seen but these generally did not persist despite continued ataluren administration. We monitored plasma adrenocorticotropic hormone and cortisol because of preclinical data in dogs suggesting that ataluren administration can be associated with adrenalitis [18]; however, no patient showed a pattern suggestive of clinical adrenocortical insufficiency. Collectively, the observed laboratory abnormalities were not clinically significant.

Long-term ( $\geqslant=25$ months), post-study safety data were available for all study participants. No notable new health conditions were reported.

\section{Pharmacokinetics}

Ataluren pharmacokinetics showed rapid drug absorption, dose-proportional differences in mean plasma exposure between dose levels, and generally steady maintenance of plasma concentrations above target trough concentrations (data not shown). The observed mean concentration values were similar to those observed in these same patients in the preceding phase 2a ataluren study [20]. 
TABLE 2 Treatment-emergent adverse events and laboratory abnormalities

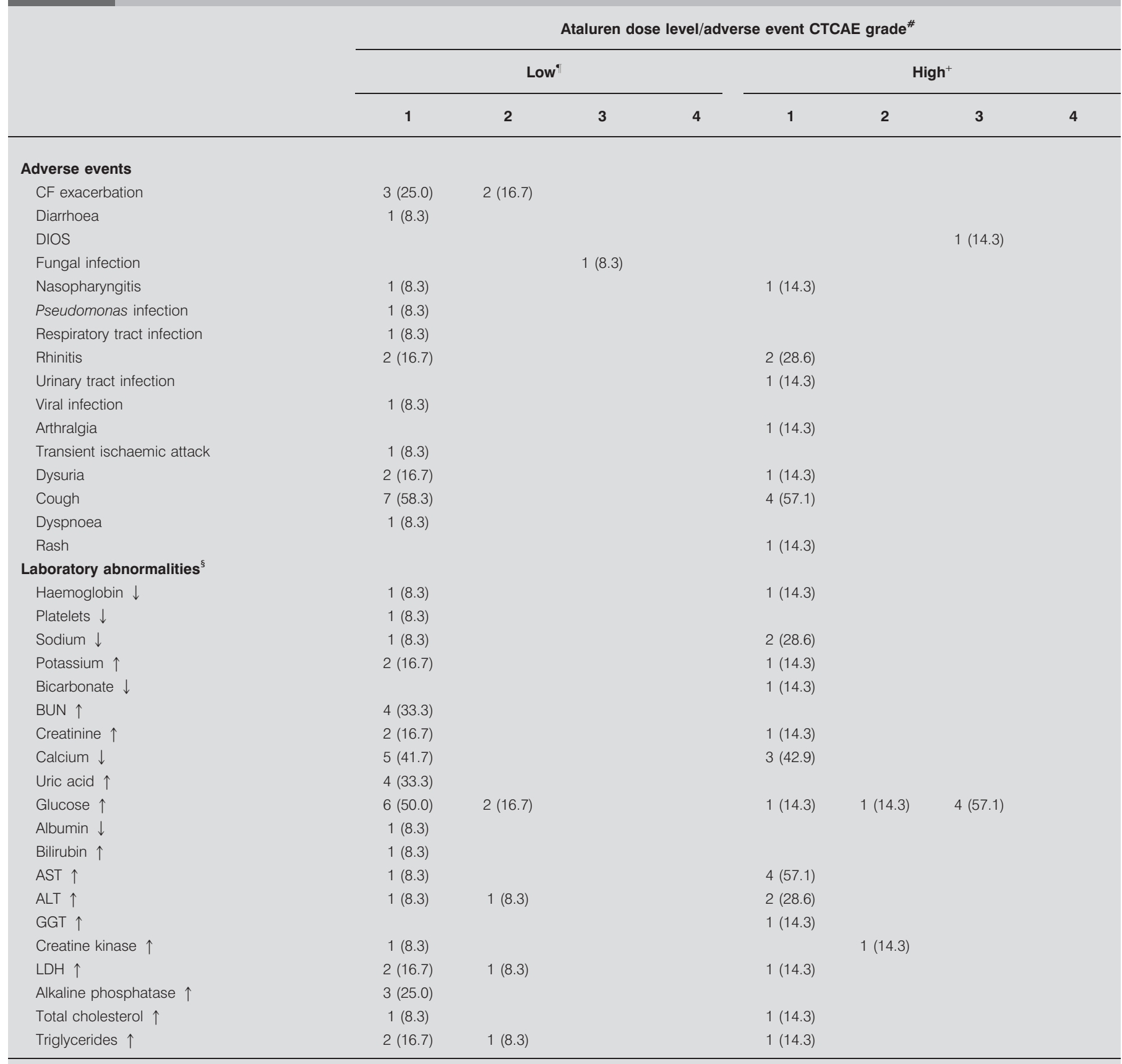

Data are presented as $\mathrm{n}(\%)$ and blank spaces in table are intentionally blank. ${ }^{*}$ : a patient with at least one occurrence of the same type of event is only listed once, by

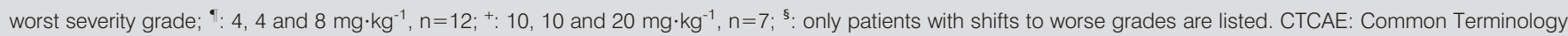
Criteria for Adverse Events; CF: cystic fibrosis; DIOS: distal ileal obstruction syndrome; $\uparrow$ : increase; $\downarrow$ : decrease; BUN: blood urea nitrogen; AST: aspartate aminotransferase; ALT: alanine aminotransferase; GGT: gamma glutamyl transferase; LDH: lactate dehydrogenase.

\section{DISCUSSION}

Despite impressive gains in prognosis through early diagnosis, aggressive supportive care and specialty care, CF remains a disabling and life-threatening disorder [2]. Treating disease manifestations persists as an important goal of drug development, but improved understanding of the molecular basis of CF has shifted the investigational focus toward therapies that address the underlying pathophysiological defect [38]. Simultaneously, progress in gene sequencing has permitted development of pharmacogenetic profiling [39]. Nonsense mutation suppression therapy with ataluren builds on these evolving paradigms. Application of ataluren constitutes a targeted therapeutic approach to genetic disease that couples genetic patient selection with a treatment designed to overcome a specific mutational abnormality. 
In a preceding phase 2a dose-ranging study in patients with nonsense mutation CF [20], we had administered ataluren in two 14-day treatment periods separated by a 14-day washout period. While pharmacological proof of concept for ataluren readthrough of premature stop codons in CFTR mRNA was observed [20], total treatment duration was limited. This extension study evaluated 12 weeks of continuous ataluren administration, the longest course of treatment yet reported in patients with CF. This trial confirms the pharmacodynamic activity of ataluren in mediating statistically significant improvements in CFTR-mediated total chloride conductance and documents the persistence of pharmacological effect over time. Trends toward improvements in pulmonary function and significant reductions in coughing, coincident with changes in ataluren-mediated CFTR activity, support the potential for clinical benefit. The longer term exposure data derived from this study support the safety profile of ataluren administration as a potential chronic therapy for nonsense mutation $\mathrm{CF}$.

Considering the short half-lives of ataluren [25] and of CFTR protein [40] and continuous epithelial cell turnover [41] during the 8- to 12-month period between the trials, no carryover effects from the end of the preceding study to the start of this extension study were expected or observed. Indeed, the mean \pm SEM change in baseline total chloride transport between the studies was only $-0.04 \pm 1.00 \mathrm{mV}(\mathrm{n}=19)$. Over this intertreatment interval, only two patients $(10.5 \%)$ had spontaneous changes of at least $-5 \mathrm{mV}(8.0$ to $-0.6 \mathrm{mV}$ and 4.0 to $-1.5 \mathrm{mV})$ and all patients had persistently abnormal total chloride transport (values less than $-5.0 \mathrm{mV}$ ). By contrast, during ataluren administration, $61 \%$ of patients experienced an ontreatment total chloride transport response (at least a $-5.0 \mathrm{mV}$ change) and $56 \%$ showed hyperpolarisation (values of at least $-5.0 \mathrm{mV}$ ), with marked improvements from uniformly abnormal values at baseline to consistently hyperpolarised values by day 84 . However, by day 112, in the absence of ataluren pharmacological effect for 28 days, all values were again abnormal, corroborating the presence of pharmacological activity during treatment. Total chloride transport improvements were noted in patients with both G542X and W1282X premature stop codon mutations, confirming data from our prior study [20] and from other ataluren trials [42], indicating that multiple nonsense mutation genotypes can be responsive to ataluren therapy. The data also demonstrate that in patients with these genotypes, amino acid substitution at the site of the nonsense mutation does not create structural disruptions of the CFTR that prevent chloride channel activity.

The time-dependent pattern of the ataluren-induced changes in total chloride transport in this study is notable. Given that responses have been observed more rapidly in other studies $[20,42]$, the reason for the progressive improvements over an extended period is not clear. Changes in pharmacokinetics were not a likely explanation for this observation when considering that mean ataluren plasma exposures were generally consistent throughout the trial. The data may indicate that ongoing cell turnover during continuous ataluren-mediated CFTR generation results in recruitment of an increasingly greater proportion of CFTR-positive epithelium. Such conjecture may be consistent with the results from in vitro studies evaluating the functional consequences associated with CFTR gene transfer [41] or the mixing of CF and non-CF cells
[43]; these experiments suggest that the magnitude of chloride transport correction is dependent not only on the amount of CFTR expressed per cell but also on the proportion of epithelial cells expressing CFTR.

Our study was not powered to document statistically significant changes in pulmonary function. However, given the available sample size, the trends toward pulmonary function improvement we did observe in the study are encouraging. It is further notable that the combined mean improvements in FEV1 and FVC occurred coincident with the greatest mean improvement in total chloride transport and that there was reversion of effect to the baseline mean upon cessation of ataluren therapy. While the open-label design mandates interpreting these data with caution, the pattern of changes is consistent with a pharmacological effect. Presumably, a timedependent, multistep process leading from pharmacological action to clinical benefit is required; putative steps may include CFTR production, recruitment of additional CFTR-expressing epithelium during mucosal turnover, increasing periciliary layer hydration, cilia mobilisation and regrowth, and decreased inflammation and infection: all ultimately resulting in reduced airway obstruction.

In patients with $\mathrm{CF}$, coughing is the most common patient complaint $[5,6]$. Chronic cough is the source of frustration, irritability and anger for patients, and often interferes with daily activities and social events [44]. Therapies designed to restore CFTR expression in epithelial cells offer the potential to increase epithelial mucous hydration and cause secondary reductions in airway irritation; a decrease in cough frequency could serve as a symptomatic integration of disease-modifying pharmacological effects. The inadequacies of relying solely upon subjective cough assessment have been long recognised [45] and guidelines for cough assessment now recommend that cough be evaluated objectively with mobile, compact, continuous recording systems that employ sensitive and specific automated cough recognition and digital data processing [46-48]. The LifeShirt device incorporates all of these attributes [29] and, as we documented in a cross-sectional evaluation of outpatient cough quantitation in patients with CF [7], substantiates very high rates of awake coughing in patients with CF. The results of longitudinal cough assessment in this study confirm that repeated 24-h cough recording is well tolerated by patients and is practical for clinic staff, resulting in excellent compliance throughout the trial. By day 84 of the study, we observed consistent reductions in mean awake cough rate (by $\sim 20 \%$ ) in both ataluren dose groups. Because the reductions occurred contemporaneously with improvements in CFTR function and pulmonary function, the cough data further supported a time-dependent ataluren-mediated effect. The disabling nature of chronic cough, the high rates of cough in patients with $\mathrm{CF}$, and these data suggesting responsiveness to therapeutic intervention all support the potential of objective cough quantitation as an important new measure of clinical benefit in studies of therapies aimed at correcting the underlying pathophysiology of CF.

The focus of this study was on observing temporal changes in study parameters; however, within the constraints of the limited sample size, we did evaluate for any substantive differences in outcome by dose. The ataluren dosing regimens 
(respective morning, midday and evening doses of either 4, 4 and $8 \mathrm{mg} \cdot \mathrm{kg}^{-1}$ or 10,10 and $20 \mathrm{mg} \cdot \mathrm{kg}^{-1}$ ) evaluated in this longer term trial were the same as those administered in our prior study [20] and in a separate short-term ataluren study in children [42]. In this trial, there was a numerically, but not a statistically greater total chloride transport at the lower dose level than at the higher dose level. These data contrast with data from a separate study in children with CF suggesting that numerically but not statistically greater activity and durability of ataluren-mediated chloride transport at the higher dose level than at the lower dose level. In this trial as in the other CF studies, the general similarity of the activity of dose levels was not likely due to similarities of exposure given that ataluren plasma concentrations increased proportional to dose. It is notable that dose selection for this trial attempted to allocate patients to the lowest dose that had been associated with the best response in the prior short-term study; because this scheme was not random, it may have resulted in assignment of the most responsive patients to the lowest dose level, thus hindering an unbiased evaluation of dose response. Given that ataluren was generally well tolerated at both dose levels, the data do not suggest a safety constraint on use of either dose level in future studies.

The data from this phase 2 study have supported the initiation of a phase 3, international, randomised, double-blind, placebocontrolled study of ataluren in $>200$ children and adults with nonsense mutation CF [49]. Based on the collective phase 2 findings from this and other studies [20,41], the dose level of 10,10 and $20 \mathrm{mg} \cdot \mathrm{kg}^{-1}$ has been selected for that trial. Given the time-dependent findings observed in this study, the phase 3 trial will assess ataluren effects over 48 weeks. The phase 3 trial is designed to evaluate change in FEV1 as the primary end-point. Major secondary clinical endpoints include exacerbation frequency and quantitative change in cough rate. Nasal NPD evaluation of change in total chloride transport is also included as an important secondary pharmacodynamic end-point in order to provide a critical link between the pharmacological action of ataluren on disease pathophysiology and the projected clinical effects of the drug on pulmonary and extrapulmonary CF manifestations.

\section{SUPPORT STATEMENT}

This work was sponsored and funded by PTC Therapeutics, Inc., which provided drug supply and study funding and was responsible for medical monitoring, data management and regulatory reporting.

\section{CLINICAL TRIAL}

This study is listed on www.clinicaltrials.gov with identifier NCT00351078.

\section{STATEMENT OF INTEREST}

Statements of interest for M. Wilchanski, L.L. Miller, D. Shoseyov, M. Cohen, S. Armoni, Y. Yaakov, T. Pugatch, M. Cohen-Cymberknoh, N.L. Miller, A. Reha, V.J. Northcutt, S. Hirawatt, K. Donnelly, G.L. Elfring, T. Ajayi and E. Kerem, and for the study itself, can be found at www. erj.ersjournals.com/site/misc/statements.xhtml

\section{ACKNOWLEDGEMENTS}

We commend the patients who have committed their time and effort to the testing of an investigational medication. Thanks are extended to M. Sinaasappel (Rotterdam, the Netherlands) for his expert review of the NPD tracings. We are grateful to A. Derchak, M. Lin and G. Delvecchio
(VivoMetrics, Ventura, CA, USA) for expert technical assistance relating to use of the LifeShirt. The technical expertise of A. Xu (SFBC Analytical Laboratories, North Wales, PA, USA) in the performance of the bioanalytical assessments is very much appreciated. We would also like to extend our gratitude to G. Elfring and J. Dancy (Innovative Analytics, Kalamazoo, MI, USA) for their expertise in data management. We are indebted to E. Kenoshi (Hadassah University Hospital, Jerusalem, Israel) and E. Colacino (EBC Clinical Monitoring, Asbury Park, NJ, USA) for capable monitoring of the conduct of this study. Manuscript support from P. Riebling (PTC Therapeutics, South Plainfield, NJ, USA) is much appreciated.

\section{REFERENCES}

1 Hanrahan JW, Wioland MA. Revisiting cystic fibrosis transmembrane conductance regulator structure and function. Proc Am Thorac Soc 2004; 1: 17-21.

2 O'Sullivan BP, Freedman SD. Cystic fibrosis. Lancet 2009; 373: 1891-1904.

3 Corey M, Edwards L, Levison $\mathrm{H}$, et al. Longitudinal analysis of pulmonary function decline in patients with cystic fibrosis. J Pediatr 1997; 131: 809-814.

4 Harrison F. Microbial ecology of the cystic fibrosis lung. Microbiology 2007; 153: 917-923.

5 Sawicki GS, Sellers DE, Robinson WM. Self-reported physical and psychological symptom burden in adults with cystic fibrosis. J Pain Symptom Manage 2008; 35: 372-380.

6 VanDevanter DR, Rasouliyan L, Murphy TM, et al. Trends in the clinical characteristics of the U.S. cystic fibrosis patient population from 1995 to 2005. Pediatr Pulmonol 2008; 43: 739-744.

7 Kerem E, Wilschanski M, Ajayi T, et al. Objective cough assessment over a 24-hour period in patients with cystic fibrosis. Pediatr Pulmonol 2009; 44: Suppl. 32, 342.

8 Kerem E, Reisman J, Corey M, et al. Prediction of mortality in patients with cystic fibrosis. N Engl J Med 1992; 326: 1187-1191.

9 Schluchter MD, Konstan MW, Davis PB. Jointly modelling the relationship between survival and pulmonary function in cystic fibrosis patients. Stat Med 2002; 21: 1271-1287.

10 Fuchs HJ, Borowitz DS, Christiansen DH, et al. Effect of aerosolized recombinant human DNase on exacerbations of respiratory symptoms and on pulmonary function in patients with cystic fibrosis. The Pulmozyme Study Group. N Engl J Med 1994; 331: 637-642.

11 Ramsey BW, Pepe MS, Quan JM, et al. Intermittent administration of inhaled tobramycin in patients with cystic fibrosis. Cystic Fibrosis Inhaled Tobramycin Study Group. N Engl J Med 1999; 340: 23-30.

12 Elkins MR, Robinson M, Rose BR, et al. A controlled trial of longterm inhaled hypertonic saline in patients with cystic fibrosis. $N$ Engl J Med 2006; 354: 229-240.

13 Bobadilla JL, Macek M Jr, Fine JP, et al. Cystic fibrosis: a worldwide analysis of CFTR mutations - correlation with incidence data and application to screening. Hum Mutat 2002; 19: 575-606.

14 Kerem B, Chiba-Falek O, Kerem E. Cystic fibrosis in Jews: frequency and mutation distribution. Genet Test 1997; 1: 35-39.

15 Shoshani T, Augarten A, Gazit E, et al. Association of a nonsense mutation (W1282X), the most common mutation in the Ashkenazi Jewish cystic fibrosis patients in Israel, with presentation of severe disease. Am J Hum Genet 1992; 50: 222-228.

16 de Gracia J, Mata F, Alvarez A, et al. Genotype-phenotype correlation for pulmonary function in cystic fibrosis. Thorax 2005; 60: 558-563.

17 McKone EF, Goss CH, Aitken ML. CFTR genotype as a predictor of prognosis in cystic fibrosis. Chest 2006; 130: 1441-1447.

18 Welch EM, Barton ER, Zhuo J, et al. PTC124 targets genetic disorders caused by nonsense mutations. Nature 2007; 447: 87-91. 
19 Du M, Liu X, Welch EM, et al. PTC124 is an orally bioavailable compound that promotes suppression of the human CFTR-G542X nonsense allele in a CF mouse model. Proc Natl Acad Sci USA 2008; 105: 2064-2069.

20 Kerem E, Hirawat S, Armoni S, et al. Effectiveness of PTC124 treatment of cystic fibrosis caused by nonsense mutations: a prospective phase II trial. Lancet 2008; 372: 719-727.

21 Wilschanski M, Armoni S, Yaakov Y, et al. PTC124 treatment over 3 months improves pharmacodynamic and clinical parameters in patients with nonsense-mutation-mediated CF. J Cystic Fibrosis 2008; 7: Suppl. 2, S22.

22 Kerem E, Yaakov Y, Armoni S, et al. PTC124 ${ }^{\mathrm{TM}}$ induces timedependent improvements in chloride conductance and clinical parameters in patients with nonsense mutation cystic fibrosis. Pediatr Pulmonol 2008; 43: Suppl. 31, 294.

23 Wilschanski M, Famini H, Strauss-Liviatan N, et al. Nasal potential difference measurements in patients with atypical cystic fibrosis. Eur Respir J 2001; 17: 1208-1215.

24 Standaert TA, Boitano L, Emerson J, et al. Standardized procedure for measurement of nasal potential difference: an outcome measure in multicenter cystic fibrosis clinical trials. Pediatr Pulmonol 2004; 37: 385-392.

25 Hirawat S, Welch EM, Elfring GL, et al. Safety, tolerability, and pharmacokinetics of PTC124, a non-aminoglycoside, nonsense mutation suppressor, following single- and multiple-dose administration to healthy male and female adult volunteers. J Clin Pharmacol 2007; 47: 430-414.

26 Schüler D, Sermet-Gaudelus I, Wilschanski M, et al. Basic protocol for transepithelial nasal potential difference measurements. J Cystic Fibrosis 2004; 3: 151-155.

27 Miller MR, Crapo R, Hankinson J, et al. General considerations for lung function testing. Eur Respir J 2005; 26: 153-161.

28 Miller MR, Hankinson J, Brusasco V, et al. Standardisation of spirometry. Eur Respir J 2005; 26: 319-338.

29 Coyle MA, Keenan DB, Henderson LS, et al. Evaluation of an ambulatory system for the quantification of cough frequency in patients with chronic obstructive pulmonary disease. Cough 2005; 1: 3 .

30 Knowles MR, Paradiso AM, Boucher AM. In vivo nasal potential difference: techniques and protocols for assessing efficacy of gene transfer in cystic fibrosis. Hum Gene Ther 1995; 6: 445.

31 Clancy JP, Bebok Z, Ruiz F, et al. Evidence that systemic gentamicin suppresses premature stop mutations in patients with cystic fibrosis. Am J Respir Crit Care Med 2001; 163: 1683-1692.

32 Sermet-Gaudelus I, Dechaux M, Vallee B, et al. Chloride transport in nasal ciliated cells of cystic fibrosis heterozygotes. Am J Respir Crit Care Med 2005; 171: 1026-1031.

33 Knudson RJ, Lebowitz MD, Holberg CJ, et al. Changes in the normal maximal expiratory flow-volume curve with growth and aging. Am Rev Respir Dis 1983; 127: 725-734.
34 Highsmith WE, Burch LH, Zhou Z, et al. A novel mutation in the cystic fibrosis gene in patients with pulmonary disease but normal sweat chloride concentrations. N Engl J Med 1994; 331: 974-980.

35 Espiritu JD, Kleinhenz ME. Paradoxical embolization in an adult patient with cystic fibrosis. Mayo Clin Proc 2000; 75: 1100-1102.

36 Sritippayawan S, MacLaughlin EF, Woo MS. Acute neurological deficits in a young adult with cystic fibrosis. Pediatr Pulmonol 2003; 35: 147-151.

37 Rao DS, Infeld MD, Stern RC, et al. Cough-induced hemiplegic migraine with impaired consciousness in cystic fibrosis. Pediatr Pulmonol 2006; 41: 171-176.

38 Jones AM, Helm JM. Emerging treatments in cystic fibrosis. Drugs 2009; 69: 1903-1910.

39 Ginsburg GS, Willard HF. Genomic and personalized medicine: foundations and applications. Transl Res 2009; 154: 277-287.

40 Swiatecka-Urban A, Brown A, Moreau-Marquis S, et al. The short apical membrane half-life of rescued $\Delta \mathrm{F} 508$-cystic fibrosis transmembrane conductance regulator (CFTR) results from accelerated endocytosis of $\triangle F 508$-CFTR in polarized human airway epithelial cells. J Biol Chem 2005; 280: 36762-36772.

41 Zhang L, Button B, Gabriel SE, et al. CFTR delivery to $25 \%$ of surface epithelial cells restores normal rates of mucus transport to human cystic fibrosis airway epithelium. PLoS Biol 2009; 7: e1000155.

42 Sermet-Gaudelus I, De Boeck K, Casimir GJ, et al. Ataluren (PTC124) induces cystic fibrosis transmembrane conductance regulator protein expression and activity in children with nonsense mutation cystic fibrosis. Am J Respir Crit Care Med 2010; 182: 1262-1272.

43 Johnson LG, Olsen JC, Sarkadi B, et al. Efficiency of gene transfer for restoration of normal airway epithelial function in cystic fibrosis. Nat Genet 1992; 2: 21-25.

44 Kuzniar TJ, Morgenthaler TI, Afessa B, et al. Chronic cough from the patient's perspective. Mayo Clin Proc 2007; 82: 56-60.

45 Kelsall A, Decalmer S, Webster D, et al. How to quantify coughing: correlations with quality of life in chronic cough. Eur Respir J 2008; 32: $175-179$.

46 Irwin RS. Assessing cough severity and efficacy of therapy in clinical research: ACCP evidence-based clinical practice guidelines. Chest 2006; 129: Suppl. 1, 232S-237S.

47 Morice AH, Fontana GA, Belvisi MG, et al. ERS guidelines on the assessment of cough. Eur Respir J 2007; 29: 1256-1276.

48 Decalmer SC, Webster D, Kelsall AA, et al. Chronic cough: how do cough reflex sensitivity and subjective assessments correlate with objective cough counts during ambulatory monitoring? Thorax 2007; 62: 329-334.

49 Riebling P. PTC Therapeutics, Inc. Study of Ataluren (PTC124) in Cystic Fibrosis. http://clinicaltrials.gov/ct2/show/NCT00803205 Date last updated: December 8, 2010. 